\title{
Hepatic abscess associated with subclinical ulcerative colitis
}

\author{
P.H. MACDONALD, MD, C.D. MERCER, MD, FRCSC, FACS
}

\begin{abstract}
Hepatic abscesses are caused by a variety of intra-abdominal inflammatory conditions, and usually result as direct extension of bacteria or of portal pyemia. A case of hepatic abscess developing prior to the acute onset of ulcerative colitis without other intra-abdominal pathology is presented. Can J Gastroenterol 1989;3(3):123-125
\end{abstract}

Key Words: Hepatic abscess, Ulcerative colitis

L'abcès hépatique associé à la rectocolite hèmorragique subclinique RESUME: Les abcès hépatiques sont causés par une variété de conditions inflammatoires intra-abdominales et résultent habituellement de l'envahissement direct des bactéries ou d'une pyémie portale. Nous présentons ici le cas d'un abcès hépatique qui s'est développé avant l'apparition des premiers signes de la rectocolite hémorragique et en l'absence d'une autre pathologie intra-adbominale.

\section{IVER ABSCESS AS A COMPLICATION OF Legional enteritis or ulcerative coli-} tis is exceedingly uncommon, especially in the latter. Presented here is a case in which the development of a liver abscess imminently preceded the first clinical sign of ulcerative colitis. The authors propose that this is a rare example of a pyogenic liver abscess associated with ulcerative colitis possibly arising from portal vein bacteremia.

\section{CASE PRESENTATION}

A 45-year-old man presented to a Kingston emergency department in July, 1985 with a two week history of spiking temperature associated with chills, rigors, lethargy and an $8 \mathrm{~kg}$ weight loss. Prior to the onset of this acute illness the patient had been completely healthy. There were no systemic or gastrointestinal symptoms to suggest active inflammatory bowel disease.
Hotel Dieu Hospital, Kingston, Ontario

Correspondence and reprints: Dr Dale C. Mercer, Assistant Professor, Department of Surgery,

Queen's University, Hotel Dieu Hospital, Kingston, Ontario K7L 5 G2

Received for publication January 3, 1989. Accepted March 8, 1989
Physical examination revealed an illlooking man with a low grade temperature of $37.5^{\circ} \mathrm{C}$. The abdomen was slightly distended and tender to palpation in the right upper quadrant, but no mass was present. The liver was not palpable. Laboratory investigations revealed the following: leucocyte count $16,900 / \mathrm{L}$ (normal 4000 to 10,000 ) with $75 \%$ polymorphonuclear leucocytes and $0 \%$ bands; hemoglobin concentration $101 \mathrm{~g} / \mathrm{L}$ (normal 130 to 180 ); erythrocyte sedimentation rate $120 \mathrm{~mm} / \mathrm{h}$ (normal 1 to 7 ); aspartate aminotransferase (AST) 39 $\mathrm{iu} / \mathrm{L}$ (normal 1 to 21 ); alanine aminotransferase (ALT) $69 \mathrm{iu} / \mathrm{L}$ (normal 1 to 30); alkaline phosphatase $259 \mathrm{iu} / \mathrm{L}$ (normal 34 to 108); and a total serum bilirubin of $10 \mu \mathrm{mol} / \mathrm{L}$ (normal 2 to 18 ). Cultures of sputum, urine and blood were all negative for pathogens.

An ultrasound scan of the abdomen showed a hyperechoic mass in the posterior segment of the right lobe of the liver. A computer assisted tomography, (CAT) scan confirmed multiple small hypodense lesions in the right lobe of the liver suggestive of multiple liver abscesses (Figure 1). No air was identified in the mass by plain films or CAT scan.

The patient underwent an exploratory laparotomy. Careful examination of the 


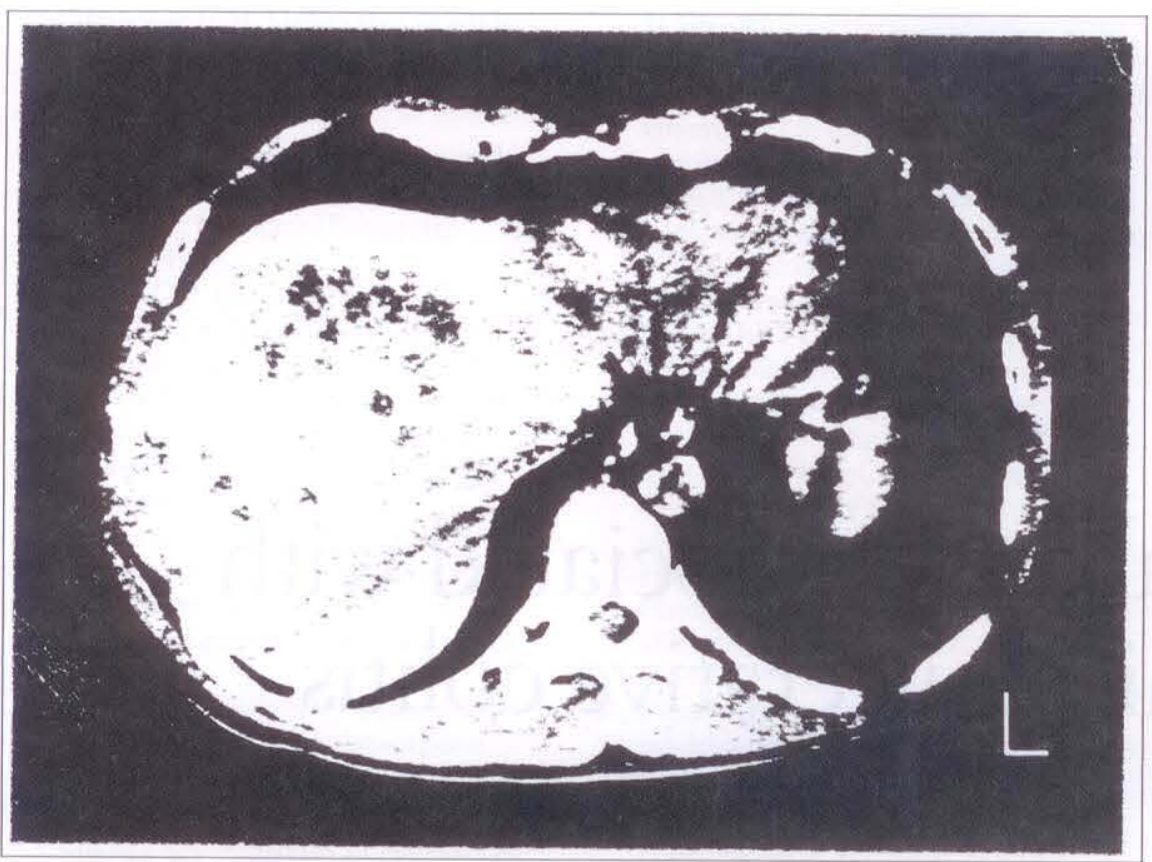

Figure 1) CAT scan demonstrates multiple hypodense areas in the right lobe of the liver compatible with a multilocuated liver abscess

intra-abdominal organs did not reveal any pathology of the appendix or the biliary tract and, aside from a few sigmoid diverticula, the findings were confined to the liver. There was no pericolonic adhesions or mesenteric thickening to suggest recent or remote episodes of diverticulitis. A large right lobe liver abscess was identified, incised and drained. Numerous smaller abscesses were found to be in continuity with the large abscess cavity. The abscess cavity contained viscous yellow-white, foul smelling pus. Stains and cultures of the abscess fluid for bacteria (aerobes and anaerobes), fungi and acid-fast bacilli were all negative. Serology for Entamoeba histolytica, using the ELISA method, was nonreactive. In spite of the negative bacteriological cultures the odour of the abscess was indicative of an anaerobic pyogenic abscess. Improper culture or isolation techniques were presumed to be the reason for failure to isolate anaerobic bacteria.

The patient's postoperative course was slow but uneventful. He was treated with intravenous metronidazole $500 \mathrm{mg}$ every $6 \mathrm{~h}$, netilmycin $80 \mathrm{mg}$ every $8 \mathrm{~h}$ and penicillin 2 million units every $4 \mathrm{~h}$. The drains were removed and the patient was discharged home two weeks after surgery, on oral metronidazole $500 \mathrm{mg}$ every $6 \mathrm{~h}$ and cephalexin $500 \mathrm{mg}$ every $6 \mathrm{~h}$ for two weeks. The patient presented again two weeks after discharge with crampy, abdominal pain and bloody diarrhea. There was no history of travel outside Ontario. A preliminary diagnosis of antibiotic induced colitis was made, however, no clinical improvement occurred within two weeks of discontinuing the antibiotics. Multiple stool cultures for bacteria, ova and parasites were all negative for pathogens. Specific cultures for enterohemorrhagic Escherichia coli were not obtained. Assays for Clostridium difficle cytotoxin were also negative.

Flexible sigmoidoscopy revealed a moderately inflamed rectum and sigmoid colon with mucosal hyperemia, granularity and friability. Histology of a rectal biopsy showed acute colitis with crypt abscesses consistent with a diagnosis of ulcerative colitis. At this point, the patient was treated with oral prednisone $40 \mathrm{mg} /$ day and sulfasalazine 2 g/day. Symptoms promptly resolved over a period of one week.

Once the acute phase of illness had subsided the remainder of the gastrointestinal tract was examined radiologically. An upper gastrointestinal series with small bowel follow through was normal. A double contrast barium enema showed only mild diverticulosis.

The patient was subsequently followed as an outpatient. He was weaned off the prednisone within six weeks and then maintained on sulfasalazine $2 \mathrm{~g} /$ day with good symptom control. A follow-up sigmoidoscopy six weeks later revealed mild erythema and friability confined to the distal $20 \mathrm{~cm}$ of the colon. Computed tomography (CT) scan of the abdomen showed no evidence of residual hepatic abscess. Complete blood count, erythrocyte sedimentation rate, AST, ALT and alkaline phosphatase were normal. One year postoperatively the patient is completely well on maintenance sulfasalazine. Sigmoidoscopy and biopsy at this time were normal.

\section{DISCUSSION}

In developed countries the majority of liver abscesses are pyogenic in origin. Only a minority are related to Entamoeba histolytica. The pathogenesis is usually related to biliary sepsis or portal vein bacteremia. However, hepatic artery bacteria and sepsis adjacent to the liver can also be causative $(1,2)$.

The incidence of portal vein bacteremia in patients with regional enteritis or ulcerative colitis is unknown. Studies of patients with ulcerative colitis have suggested an increased incidence of portal bacteremia. However, the significance of this has not yet been determined $(3,4)$. In spite of this possible increased incidence of portal bacteremia, the literature contains very few reports of liver abscess associated with regional enteritis or ulcerative colitis (5-8). In fact, many authors do not even cite these two conditions when discussing the possible etiology of hepatic abscess. Lansburg et al (6) reported only one case of hepatic abscess among 1333 patients with ulcerative colitis and this was in a patient with very severe disease. Thus, evidence to date suggests that liver abscess as a complication of inflammatory bowel disease is a rare phenomenon.

The development of a liver abscess in patients with inflammatory bowel disease can be due to suppurative pylephlebitis $(9,10)$, a fistulization into the biliary tree (11) or direct extension of bacteria from an adjacent subhepatic abscess. The latter two occur only with Crohn's 
disease while the former can occur with ulcerative colitis (3).

The present patient is unusual in that the hepatic abscess preceded the first clinical sign of ulcerative colitis. Thorough review of any antecendent history of similar large bowel symptoms elicited one episode lasting two days of watery nonbloody diarrhea seven years previously which resolved without diagnosis or treatment. This may represent an early episode of 'subclinical' inflammatory bowel disease but with no other manifestations for seven years this seems less likely. It is impossible to prove the exact etiology of the present patient's liver abscess. However, the only other operative pathology found was diverticulosis. At no time had the patient had signs or symptoms to suggest diverticulitis and the double contrast barium enema showed the diverticular disease to be quite mild. Thus, in view of the more active disease process of ulcerative colitis, it is felt that the diverticulosis was unrelated to the hepatic abscess.

The fact that this patient's abscess appeared to be sterile is not an uncommon finding in a review of pyogenic hepatic abscesses by McDonald and colleagues

\section{REFERENCES}

1. Sleisenger MH, Fordtran JS. Gastrointestinal Disease Pathophysiology, Diagnosis, Management, 3rd edn. Philadelphia: W.B. Saunders Company. 1983.

2. Balasegram M. Management of hepatic abscess. Curr Prob Surg 1981; 18:285-340.

3. Brooke BN, Slaney G. Portal bacteremia in ulcerative colitis. Lancet 1958;i:1206-7.

4. Eade MD, Brooke BN. Portal bacteremia in cases of ulcerative colitis submitted to colectomy. Lancet 1959; : 1008-9.

5. Nelson A, Frank HD, Taubin HL. Liver abscess - A complication of regional enteritis. Am J Gastroenterol 1979:72:282-4.

6. Lansbury J. Bargen JA. The association of multiple hepatic abscesses and chronic
(12), pathogens could not be isolated in at least $7 \%$ of cases. The pathogen in these cases was presumed to have been an anaerobe. Sabbaj(13) suggested that only $25 \%$ of anaerobes are recovered from hepatic abscesses with the most commonly isolated being a Gram-positive cocci. This poor microbiologic isolation of anaerobic bacteria is often due to improper handling of samples and poor culturing techniques. Specific series suggest a high frequency of isolation of anaerobes when samples are handled adequately and stringent anaerobic culture techniques are implemented (14-16).

Amoebic hepatic abscess must always be excluded and serology is an excellent discriminator in distinguishing between amoebic and pyogenic abscesses. The sensitivity of serology is greater than $95 \%$ and even higher with invasive amoebiasis $(1,16)$. In the present patient the serology was nonreactive and Entamoeba histolytica was not isolated from the stool. This eliminates the possibility of amoebic hepatic abscess.

Treatment of pyogenic liver abscess consists of adequate drainage plus appropriate antibiotic coverage. CT scan guided percutaneous drainage is at times

ulcerative colitis. Med Clin North Am 1933; 16:1427-31.

7. Crass JR. Liver abscess as a complication of regional enteritis - Interventional consideration. Am J Gastroenterol 1983;78:747-9.

8. de la Maza LM, Naeim F, Berman LD. The changing etiology of liver abscess. IAMA 1974;227:161-3.

9. Taylor FW. Regional enteritis complicated by pylephlebitis and multiple liver abscess. Am J Med 1949;7:838-40.

10. Lerman B, Garlack JH. Janaivitz HD. Suppurative pylephlebitis with multiple liver abscesses complicating regional enteritis. Ann Surg 1962;155:441-8.

11. Zarnow H, Grand TH, Spellberg M, et al. Unusual complications of regional enteritis - Duodenobiliary fistula and hepatic appropriate for a unilocular hepatic abscess. For two reasons this alternative was not chosen. The abscess appeared to be multiloculated (confirmed at surgery) and the etiology of the abscess was not apparent. Laparotomy to efficiently break down all loculations and place multiple drains and to evaluate and treat other intra-abdominal pathology was judged most efficacious.

Resolution of a liver abscess should be determined by serial ultrasounds or CT scans. Sinograms into the abscess cavity via the drain site is also an effective technique to identify shrinkage of the cavity as the volume of drainage decreases. Best estimates of proper duration of antibiotic therapy in resolving cases are speculative but a period of four to six weeks has been recommended (17).

It is conceivable that the present patient had two independent metachronous disease processes, namely hepatic abscess and ulcerative colitis. However, their relationship in time and lack of other pathology suggests a correlation. The authors, therefore, propose that this patient represents a rare case of hepatic abscess associated with ulcerative colitis.

abscess. JAMA 1976;235:1880-1.

12. McDonald AP, Howard RJ. Pyogenic liver abscess. World J Surg 1980:4:3679-80.

13. Sabbaj J. Anaerobes in liver abscess. Rev Infect Dis 1984; 6(Suppl 1):S152-6.

14. Svenson RM, Larber B, Michaelson TC, Spaulding $\mathrm{EH}$. The bacteriology of intraabdominal infections. Arch Surg 1974:109:398-9.

15. Eykyn S, Phillips I. Pyogenic liver abscess. Br Med J 1980;280:1617.

16. Conter RL, Pitt HA, Tompkins RK, Longmire WP. Differentiation of pyogenic from amebic hepatic abscesses. Surg Gynecol Obstet 1986;162:114-20.

17. Pitt HA, Zuidema GD. Factors influencing mortality in treatment of pyogenic hepatic abscesses. Surg Gynecol Obstet 1975;140:228. 


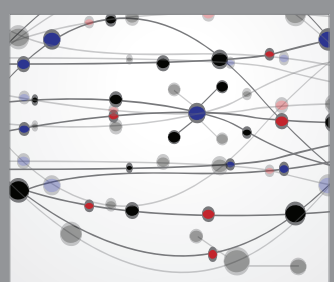

The Scientific World Journal
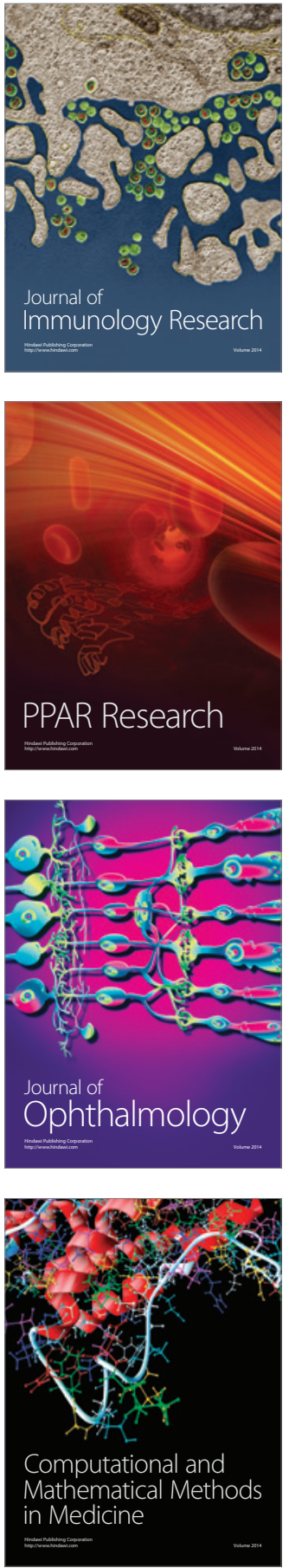

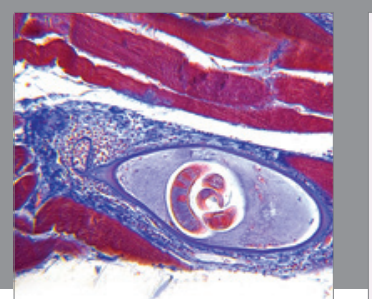

Gastroenterology Research and Practice

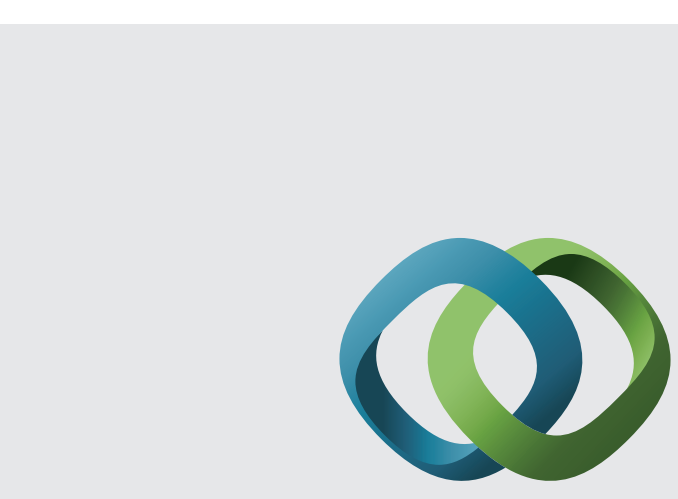

\section{Hindawi}

Submit your manuscripts at

http://www.hindawi.com
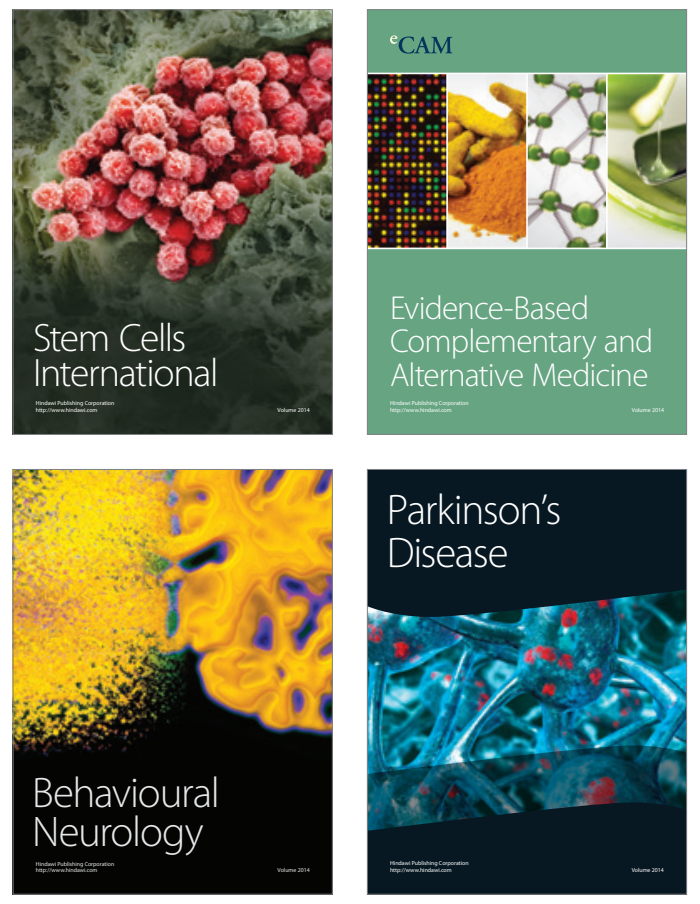
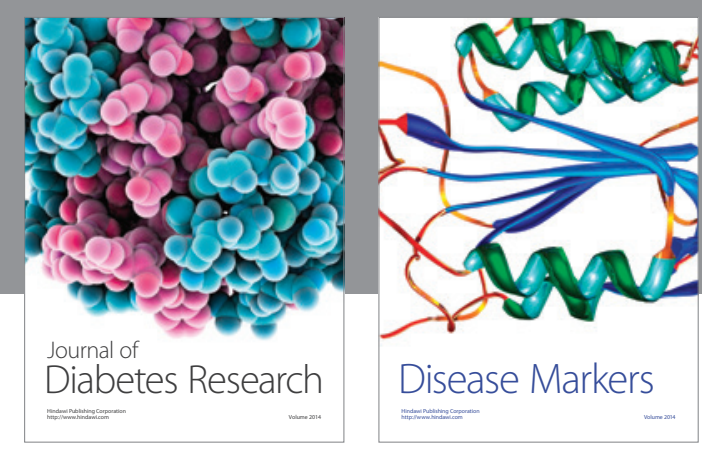

Disease Markers
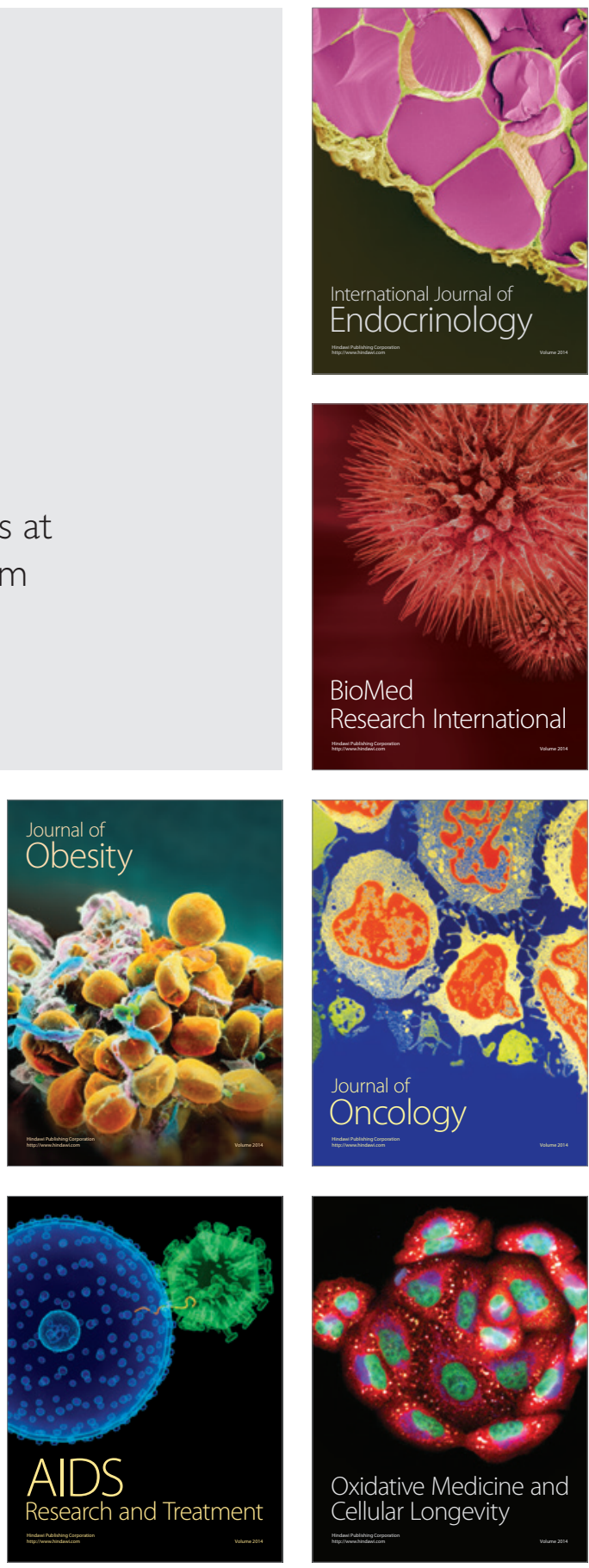